\title{
Análise da produção científica sobre os serviços farmacêuticos comunitários no enfrentamento da pandemia pelo coronavírus
}

\author{
Analysis of scientific production on community pharmaceutical \\ services in confronting the coronavirus pandemic
}

Nília Maria de Brito Lima Prado', Vinícius Nunes Carvalho', Fabiely Gomes Nunes', Naila Neves de Jesus ${ }^{\mathbf{2}}$, Hebert Luan Pereira Campos dos Santos' ${ }^{\mathbf{1}}$, Adriano Maia dos Santos ${ }^{\mathbf{1}}$

DOI: 10.1590/0103-1104202112921

RESUMO Esta revisão buscou sintetizar as práticas em serviços farmacêuticos relacionadas ao gerenciamento e às atividades clínicas em distintos contextos para a prevenção e a assistência durante a epidemia da Covid-19. Trata-se de uma revisão da literatura sobre políticas públicas de saúde para a reorganização da assistência farmacêutica em países atingidos pela Covid-19 realizada nas bases de dados Web of Science, Science Direct e Biblioteca Virtual em Saúde. Foram selecionados 9 artigos, sendo todos publicados em 2020. Destes, 90\% foram publicados pelo periódico ‘Research in Social an Administrative Pharmacy' e tiveram como local de realização de estudo países de três continentes. Foram relatadas e debatidas experiências sobre a atuação do profissional farmacêutico e o papel da farmácia comunitária no contexto da pandemia da Covid-19, e sobre a reorganização destes serviços, evidenciadas no contexto de crise, tanto no que tange à oferta de serviços quanto ao próprio espraiamento da doença. O modelo adotado pelos países para o enfrentamento da Covid-19, no âmbito dos serviços farmacêuticos comunitários, baseou-se em atividades para a garantia da qualidade e logística de insumos e medicamentos, triagem e monitoramento de pacientes, acompanhamento farmacoterapêutico, educação em saúde e informações sobre o uso de medicamentos.

PALAVRAS-CHAVE Assistência farmacêutica. Serviços comunitários de farmácia. Infecções por coronavírus. Acesso universal aos serviços de saúde.

\begin{abstract}
This review sought to synthesize the practices in pharmaceutical services related to management and clinical activities in different contexts for prevention and assistance during the Covid-19 epidemic. This is a review of the literature on public health policies for the reorganization of pharmaceutical assistance in countries affected by Covid-19 carried out in the Web of Science, Science Direct and Virtual Health Library databases. 9 articles were selected, all published in 2020. Of these, 90\% of the articles were published by the journal 'Research in Social an Administrative Pharmacy' and had countries of three continents as study sites. Experiences about the performance of the pharmaceutical professional and the role of the community pharmacy in the context of the Covid-19 pandemic, and about the reorganization of these services, evidenced in the context of crisis, were reported and debated, both with regard to the provision of services and the spread of the disease itself. The model adopted by countries to confront Covid-19, within the scope of community pharmaceutical services, was based on activities for quality assurance and logistics of supplies and medicines, screening and monitoring of patients, pharmacotherapeutic accompaniment, health education and information on medication use.
\end{abstract}

Bahia (UFBA) - Vitória da

Conquista (BA), Brasil.

hebertluanvph@hotmail.com

2 Universidade Estadual do Sudoeste da Bahia (Uesb) - Vitória da Conquista (BA), Brasil.
KEYWORDS Pharmaceutical services. Community pharmacy services. Coronavirus infections. Universal access to health care services. 


\section{Introdução}

No âmbito da infecção pelo novo coronavírus (Sars-CoV-2), da pandemia da Covid-19, estão sendo desenvolvidas medidas de saúde pública, de acordo com a fase de resposta à propagação do vírus. O sucesso das medidas preventivas depende essencialmente da colaboração dos cidadãos e das instituições. É importante incentivar e salvaguardar o papel específico dos estabelecimentos, nomeadamente aqueles que garantem as condições para o tratamento da Covid-19 nos sistemas de saúde.

A Organização Mundial da Saúde (OMS)2 ao reconhecer a fragilidade de muitos sistemas de saúde do mundo, propôs diretrizes para os países manterem serviços essenciais de saúde durante todo o período da pandemia, que incluem as farmácias comunitárias, para oferecer aconselhamento essencial, fornecimento de informações e medicamentos a pacientes e comunidades locais. Pelo papel fulcral que representam no sistema de saúde, e pelas especificidades da população a que servem, as farmácias devem, ainda, adotar uma série de medidas que visem a proteger os seus colaboradores e cidadãos, bem como contribuir para impedir a propagação da Covid-1932.

O papel dos cuidados farmacêuticos é significativo para pessoas com doenças crônicas e é particularmente relevante durante esse período de pandemia da Covid-19, em que muitas pessoas têm várias preocupações relacionadas ao uso de medicamentos. Os serviços farmacêuticos estabeleceram as práticas de gerenciamento do fornecimento e da assistência farmacêutica sob quatro aspectos: pessoal, gerenciamento de suprimento de medicamentos, gerenciamento de uso de medicamentos offlabel e atendimento para a adesão ao tratamento farmacoterapêutico ${ }^{4}$.

Para o tratamento de Covid-19, farmacêuticos realizam o monitoramento de reações adversas e participam de consulta multidisciplinar em equipes de saúde, reconciliação de medicamentos e revisão de prescrições, promovendo o uso racional de medicamentos, monitoramento farmacoterapêutico e gerenciamento de riscos de medicamentos, contribuindo para identificar casos suspeitos e evitar uma maior disseminação na comunidade ${ }^{5}$.

Nesse sentido, a farmácia comunitária é um dos estabelecimentos de saúde que tem um papel fundamental a desempenhar no controle da pandemia atual. Como o escopo da prática farmacêutica comunitária varia consideravelmente entre os países, é importante examinar os modelos de assistência farmacêutica que podem contribuir com a resposta de saúde pública ou privada à Covid-19 e manter a continuidade dos serviços farmacêuticos alinhada às determinações e às práticas das equipes e dos serviços de saúde ${ }^{6}$.

Este artigo sintetizou as práticas em serviços farmacêuticos relacionadas ao gerenciamento e às atividades clínicas em distintos contextos para a prevenção e a assistência durante a epidemia da Covid-19. Essas informações podem contribuir para a tomada de decisões futuras sobre a reestruturação dos serviços de saúde por gestores e formuladores de políticas públicas em resposta à crise, como a pandemia de Covid-19.

\section{Aspectos metodológicos}

Trata-se de uma revisão sistemática da literatura sobre políticas públicas de saúde para a reorganização da assistência farmacêutica em países atingidos pela pandemia do novo coronavírus.

Revisores independentes realizaram a busca de trabalhos nas bases de dados Web of Science, Science Direct e Biblioteca Virtual em Saúde (BVS), utilizando as seguintes combinações de palavras-chave: Assistência Farmacêutica; Serviços Comunitários de Farmácia; Infecções por Coronavírus; Sistemas de Saúde. A coleta foi realizada entre 01 a 15 de abril de 2020.

Adotou-se como critério de inclusão os estudos que abordavam políticas públicas de assistência farmacêutica, os aspectos relacionados à organização dos serviços e os desafios 
para sua implementação em países atingidos pela pandemia da Covid-19. Não houve exclusão, a priori, de nenhuma abordagem metodológica, tendo sido incluídos tanto artigos qualitativos quanto quantitativos. $\mathrm{O}$ estudo incluiu artigos escritos em inglês.

Os motivos para a exclusão dos artigos foram a ausência da análise e discussão de aspectos relacionados estritamente a políticas e organização de serviços farmacêuticos para enfrentamento do coronavírus.

Iniciou-se a seleção dos estudos por meio da leitura dos títulos e dos resumos, observando-se os critérios de inclusão. Os artigos selecionados foram lidos na íntegra. Foi estruturada uma planilha do Excel $^{\circledR}$ contendo os seguintes termos: autores, ano de publicação, país do estudo, desenho do estudo/metodologia, número de pessoas investigadas, objetivos, população estudada, política pública farmacêutica, ações para enfrentamento do coronavírus e principais resultados.

A sistematização envolveu as etapas de identificação, fichamento, análise e interpretação de resultados de estudos selecionados. Essa revisão seguiu as recomendações da Prisma
(Preferred Reporting Items for Systematic Reviews and Meta-Analyses) ${ }^{7}$.

Os artigos identificados nas três bases de dados foram organizados no software Microsoft Excel ${ }^{\circledR}$ 2007, considerando as seguintes variáveis: ano da publicação, título, nome dos autores, periódico em que foi publicado, desenho/estratégia de estudo e principais achados, sendo esses dois últimos definidos inicialmente a partir do resumo do artigo, confirmados pela leitura do artigo na íntegra, se necessário.

Os artigos foram inicialmente analisados a partir de seus títulos, seguidos da leitura de seus resumos e posterior leitura dinâmica na íntegra. Inicialmente, foram selecionadas 22 publicações, sendo 8 na Biblioteca Virtual em Saúde (BVS), 8 na Science Direct e 6 na Web of Science. Após a leitura de títulos e resumos, foram excluídos 4 na BVS e 1 na Web of Science, por se tratar de assistência farmacêutica no âmbito hospitalar, 4 na Science Direct e 4 na Web of Science, ambos por duplicação. Após a leitura na íntegra, foram obtidos 9 artigos, que compuseram o universo a ser analisado (figura 1).

Figura 1. Busca, seleção, inclusão e exclusão de estudos sobre políticas públicas de saúde pública para a reorganização da assistência farmacêutica em países atingidos pela pandemia do coronavírus

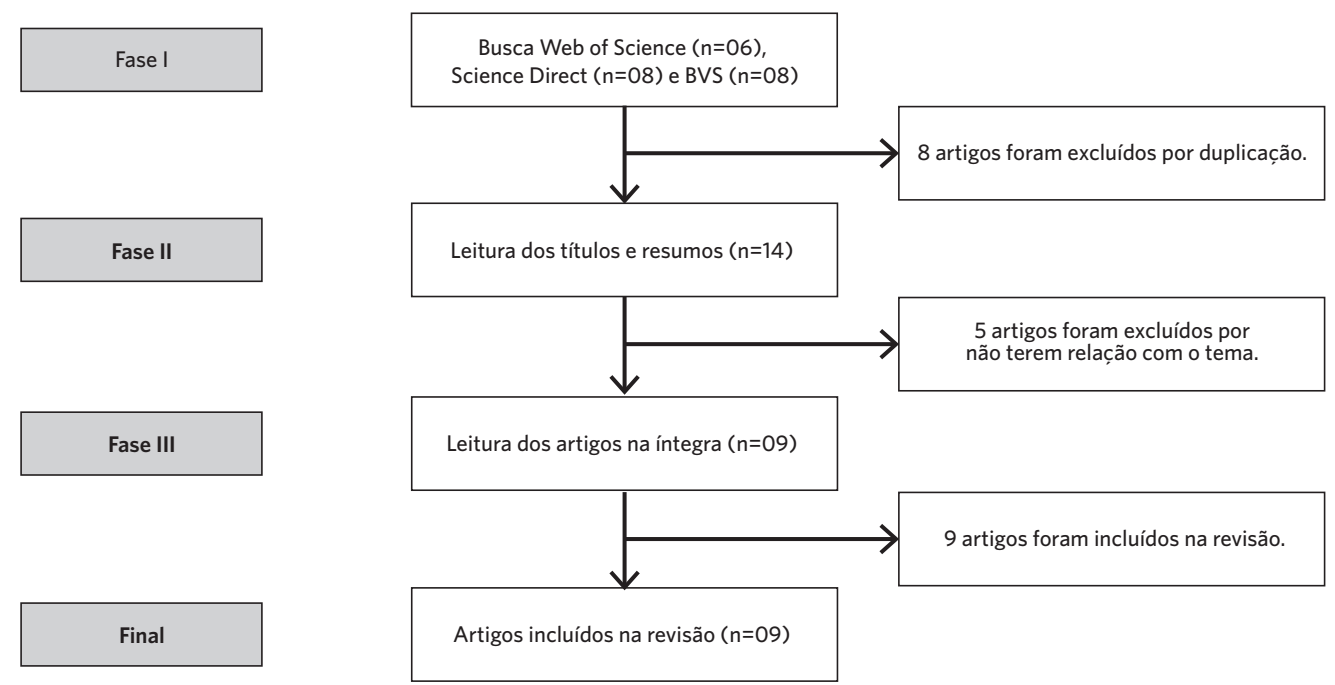




\section{Resultados e discussão}

Dos 09 artigos selecionados, 08 foram publicados na revista 'Research in Social an Administrative Pharmacy' e 01 artigo foi publicado no 'International Journal of Clinical Pharmacy', sendo a origem geográfica bem diversa: artigos do continente asiático (02 da China, 01 de Macau e 01 dos Emirados Árabes), artigos provenientes do continente americano (dos Estados Unidos e da Colômbia), artigos originários do continente africano, (da Nigéria e de Gana). Todas as produções tinham natureza qualitativa e concentraram-se no ano de 2020 em vista da atualidade e da urgência da temática no cenário vigente.

Os artigos propuseram, de maneira geral, expor experiências e suporte científico para a atuação do profissional farmacêutico, debater o papel da farmácia comunitária no contexto da pandemia da Covid-19 e como se deu a reorganização dos serviços para o fornecimento do cuidado ao paciente (quadro 1).

Quadro 1. Estratégias adotadas por cada país para a reorientação dos serviços farmacêuticos comunitários em países selecionados

\begin{tabular}{|c|c|c|c|c|}
\hline Autores & $\begin{array}{l}\text { País de } \\
\text { origem }\end{array}$ & $\begin{array}{l}\text { Política } \\
\text { Farmacêutica }\end{array}$ & Estratégias adotadas & Âmbito \\
\hline $\begin{array}{l}\text { Zheng et } \\
\text { al. } 8\end{array}$ & China & $\begin{array}{l}\text { Assistência Farma- } \\
\text { cêutica e Atenção } \\
\text { Farmacêutica }\end{array}$ & $\begin{array}{l}\text { - As farmácias comunitárias devem possuir suprimento adequado de medicamentos } \\
\text { e produtos preventivos para Covid-19 (máscaras, álcool em gel); - Fornecimento de } \\
\text { informações em tempo real para pacientes portadores de doenças crônicas através de } \\
\text { websites da farmácia ou aplicativos de celular informando-os quando necessitam comprar } \\
\text { seus medicamentos ou para tirar dúvidas sobre o seu uso; - Serviço de entrega em domi- } \\
\text { cílio; - As farmácias chinesas fizeram parcerias com os comitês dos bairros para fornecer } \\
\text { serviços de entregas de medicamentos em comunidades em quarentena; - Para pacien- } \\
\text { tes com doenças específicas, como câncer, hepatite e síndrome do intestino irritável, as } \\
\text { farmácias trabalharam com as empresas farmacêuticas para garantir o fornecimento dos } \\
\text { medicamentos durante a pandemia; - Fornecimento de cuidados farmacêuticos com a fi- } \\
\text { nalidade de promover a prevenção e o controle da Covid-19 e a garantia do uso seguro de } \\
\text { medicamentos pelo paciente.; - As consultas e orientações podem ser feitas através de } \\
\text { telefone, aplicativos e internet; - Divulgação do acesso remoto a farmacêuticos através } \\
\text { de pôsteres ou folhetos na farmácia e nas comunidades vizinhas, notificações na internet, } \\
\text { mensagens de texto ou e-mails. }\end{array}$ & Privado \\
\hline Liu et al.1 & China & $\begin{array}{l}\text { Assistência Farma- } \\
\text { cêutica e Atenção } \\
\text { Farmacêutica }\end{array}$ & $\begin{array}{l}\text { - Fornecimento de medicamentos preventivos e de tratamento, prestação cuidados } \\
\text { farmacêuticos; - Trabalho em conjunto com outras organizações de saúde, profissionais, } \\
\text { agências governamentais para atender à necessidade de serviços, tais como: garantir } \\
\text { suprimento, armazenamento e transporte adequados dos medicamentos, redigir guias de } \\
\text { orientações de serviços profissionais aos farmacêuticos e farmácias, estabelecimento de } \\
\text { serviços remotos de farmácia, educação do público com foco na prevenção, entre outros; } \\
\text { - Estabelecer formulários terapêuticos com medicamentos de emergência baseados em } \\
\text { guias de tratamento; - Participação do farmacêutico em ensaios clínicos de avaliação de } \\
\text { medicamentos. }\end{array}$ & Público \\
\hline Ung, C.9 & Macau & $\begin{array}{l}\text { Assistência Farma- } \\
\text { cêutica e Atenção } \\
\text { Farmacêutica }\end{array}$ & $\begin{array}{l}\text { - Implementação de práticas de prevenção e controle de infecção na farmácia comunitá- } \\
\text { ria; - Garantir a segurança pessoal, reduzir os riscos de transmitir o vírus entre as pesso- } \\
\text { as e impedir a propagação de infecção através do contato de itens na farmácia; - Prática } \\
\text { regular e correta de higiene entre todos os funcionários e garantia de que toda a estrutura } \\
\text { de proteção seja instalada e que os produtos de higiene das mãos estejam prontamente } \\
\text { disponíveis para uso; - Limpeza regular de superfícies e itens; - A equipe de farmácia } \\
\text { deve ser incentivada a educar todos os clientes sobre estratégias de higiene das mãos, } \\
\text { prevenção e controle de infecções; - Farmacêuticos devem reforçar medidas de higiene } \\
\text { pessoal e ambiental do público; - Familiarização do farmacêutico com as formulações à } \\
\text { base de álcool para uma possível produção de álcool gel na farmácia comunitária, a fim } \\
\text { de suprir a demanda por esses produtos; Orientação quanto ao uso de }\end{array}$ & $\begin{array}{l}\text { Público } \\
\text { /priva- } \\
\text { do }\end{array}$ \\
\hline
\end{tabular}


Quadro 1. (cont.)

\begin{tabular}{lll}
\hline Autores & $\begin{array}{l}\text { País de } \\
\text { origem }\end{array}$ & $\begin{array}{l}\text { Política } \\
\text { Farmacêutica }\end{array}$ \\
\hline & & \\
& & \\
& & \\
& & \\
& & \\
Aruru, & EUA & - Preparação e \\
Truong, & & resposta a emer- \\
Clark10 & gências (Emergency \\
& & Prepare \& Response \\
& & - EP\&R);- Assistên- \\
& cia Farmacêutica.
\end{tabular}

Estratégias adotadas

máscaras aos consumidores, ajudando-os a diferenciar máscaras cirúrgicas de outros tipos de máscaras faciais não recomendadas para a proteção contra o vírus; - Controlar o preço das máscaras cirúrgicas dentro da faixa de preço razoável e ajudar a implementar o 'The Guaranteed Mask Supply for Macao Residents Scheme' em resposta à nova política do governo; - Aconselhar o consumidor sobre a escolha correta da máscara para sua proteção individual;- O governo local comprou 20 milhões de máscaras e anunciou a distribuição controlada através das farmácias; - O governo criou uma página da web com atualizações a cada 15 minutos do número de máscaras disponíveis em diferentes farmácias e seus detalhes de contato; - Parceria-público privada entre governo e farmácias comunitárias para dispensação e cuidados farmacêuticos para pacientes dos Institutos de Saúde Pública; - Monitoramento do estoque de produtos farmacêuticos através de um sistema de dados de compra e venda desses produtos para monitoramento em tempo real do estoque.

- Remoção de barreiras para suprir a falta de produtos médicos e fornecer garantias para a continuidade do atendimento; - Facilitar barreiras operacionais para resolver problemas de força e fluxo de trabalho para farmacêuticos durante um período de crise; - Expansão do papel dos profissionais de farmácia e farmácias durante a pandemia com a incorporação de recurso e educação EP\&R em seus sites sobre o Covid-19; - Serviços de farmácia considerados essenciais permaneceram abertos para fornecer acesso a medicamentos, produtos e suprimentos e saúde. Muitas farmácias estão expandido serviços, aplicando isenção de taxas de entrega, extensão de horas e extensão de períodos de recarga; - Profissionais de farmácia devem estar preparados para gerenciar medicamentos e suprimentos médicos enquanto trabalham em equipes de saúde integradas; - Os farmacêuticos devem continuar a imunizar rotineiramente o público e incorporar imunizações adicionais em emergências, assim que estiverem disponíveis; - Permissão da FDA de uso de produtos médicos não aprovados ou usos não aprovados de produtos médicos já validados; - Estabelecimento de pontos de dispensação e administração de MCMs (medical countermeasures); - Criação de abrigos de emergência com o fornecimento de serviços essenciais; - O farmacêutico está autorizado a testar, tratar e imunizar pacientes ou a comunidade, especialmente em áreas de acesso à saúde limitado; - Os farmacêuticos devem continuar a monitorar e relatar efeitos adversos relacionados a medicamentos e vacinas recentemente aprovados para o uso em emergências; - Educação farmacêutica no contexto de saúde pública e EP\&R; - Pesquisa e disseminação de impactos e resultados de EP\&R para o reconhecimento da contribuição dos profissionais de farmácia durante emergências de saúde pública.

Carico Jr, EUA Atenção Farma-

Sheppard,

Thomas ${ }^{15}$

Hedimaa, Adeyemib, Ikunaiyec 11

Nigéria Assistência Farmacêutica e Atenção Farmacêutica

Amarilis et Colôm- Atenção Farmaal. $\mathbf{1 4}$ bia cêutica
Uso do modelo Health Belief Model como um guia de comunicação por farmacêuticos comunitários para ajudar na adesão de medidas preventivas de mitigação da transmissão do vírus e motivar a mudança de comportamento.

- Garantir o suprimento de medicamentos e produtos farmacêuticos necessários; - As farmácias podem compartilhar as informações de medicamentos disponíveis no estoque através do seu site ou por um aplicativo de celular, além de informar ao paciente através desses canais quando há necessidade de comprar medicamentos; - Para pacientes que não podem ir à farmácia, disponibilizar pedido por e-mail, entrega em domicílio; - Autorização para o farmacêutico realizar testes rápidos; - Farmacêuticos devem prover imunização (quando disponível) para Covid-19 na farmácia comunitária; - Promover o uso seguro e racional dos medicamentos.

Desenvolvimento de uma proposta de fluxograma de sinais e sintomas para que o farmacêutico possa contribuir para a detecção precoce e o encaminhamento adequado de casos suspeitos de Covid-19.
Âmbito

Privado

Não identificado

Não identificado

Não identificado 
Quadro 1. (cont.)

\begin{tabular}{|c|c|c|c|c|}
\hline Autores & $\begin{array}{l}\text { País de } \\
\text { origem }\end{array}$ & $\begin{array}{l}\text { Política } \\
\text { Farmacêutica }\end{array}$ & Estratégias adotadas & Âmbito \\
\hline $\begin{array}{l}\text { Al-Qutei- } \\
\text { mat, Amer12 }\end{array}$ & $\begin{array}{l}\text { Emi- } \\
\text { rados } \\
\text { Árabes }\end{array}$ & $\begin{array}{l}\text { Assistência Farma- } \\
\text { cêutica e Atenção } \\
\text { Farmacêutica }\end{array}$ & $\begin{array}{l}\text { Os farmacêuticos devem aconselhar as pessoas não suspeitas de Covid-19 a praticar o } \\
\text { distanciamento social, evitar espaços fechados e lotados; - Informá-las para manterem } \\
\text { uma distância de pelo menos } 2 \text { metros de qualquer indivíduo suspeito de ter Covid-19; - } \\
\text { Incentivar a prática de higiene das mãos, demonstrar maneiras mais seguras e educadas } \\
\text { de tossir e espirrar; - Garantir o reabastecimento de medicamentos, obtendo substi- } \\
\text { tuições seguras; - Prescrever medicamentos de venda livre para algumas indicações, } \\
\text { reduzindo visitas hospitalares desnecessárias; - Oferta de entrega em domicílio de medi- } \\
\text { camentos e produtos, consultas farmacêuticas on-line ou por telefone. }\end{array}$ & $\begin{array}{l}\text { Não } \\
\text { identi- } \\
\text { ficado }\end{array}$ \\
\hline $\begin{array}{l}\text { Kretchy et } \\
\text { al. }{ }^{\mathbf{6}}\end{array}$ & Gana & $\begin{array}{l}\text { Atenção Farma- } \\
\text { cêutica }\end{array}$ & $\begin{array}{l}\text { - Cuidado Farmacêutico focado na prevenção, identificação e/ou resolução de problemas } \\
\text { relacionados ao uso de medicamentos para os clientes de seu estabelecimento; - Pro- } \\
\text { moção do uso racional de medicamentos, principalmente para pessoas que apresentam } \\
\text { doenças crônicas; - Provisão de informação sobre medicamentos, aconselhamento sobre } \\
\text { medicamentos utilizados em doenças crônicas; - Promover adesão e prover serviços de } \\
\text { acompanhamento; - Disponibilizar estoque de medicamentos essenciais; - Prover infor- } \\
\text { mação adequada sobre medidas de prevenção e supressão da transmissão; - Identificar } \\
\text { pacientes suspeitos através de triagem baseada em protocolos; - Promover adesão ao } \\
\text { medicamento; - Revisão da medicação e acompanhamento do paciente. }\end{array}$ & $\begin{array}{l}\text { Não } \\
\text { identi- } \\
\text { ficado }\end{array}$ \\
\hline
\end{tabular}

Fonte: Elaboração própria.

Durante a pandemia, os profissionais de farmácia têm respondido altamente à crise. As farmácias comunitárias fornecem serviços essenciais aos pacientes e às equipes de saúde por meio do provimento contínuo de medicamentos e tratamento, e continuam abertas - e, em algumas áreas, oferecem horários e serviços prolongados -, para fornecer acesso crítico a medicamentos, produtos e suprimentos de saúde. Em paralelo, esses serviços devem estar preparados para gerenciar medicamentos e suprimentos médicos, enquanto atendem a equipes de saúde integradas e equipes comunitárias em resposta a situações de emergência ${ }^{6}$ (figura 2). 
Figura 2. Modelo de assistência farmacêutica na Atenção Primária à Saúde para enfrentamento da pandemia de Covid-19 nos países incluídos no estudo

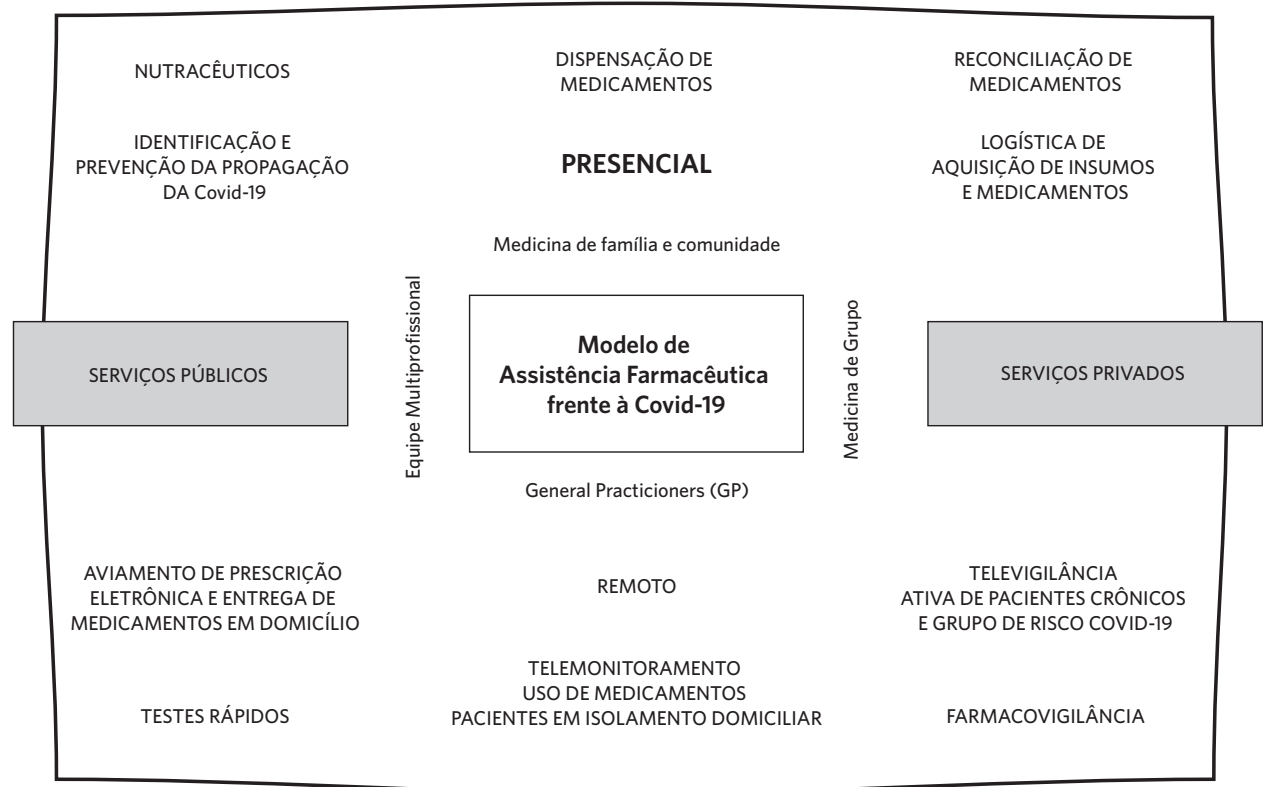

Fonte: Elaboração própria.

As estratégias adotadas para o enfrentamento da Covid-19, no âmbito dos serviços farmacêuticos comunitários, basearam-se em políticas farmacêuticas já estabelecidas previamente nos países sob as premissas da assistência farmacêutica ${ }^{\mathbf{1}, \mathbf{8}-12}$, que envolvem as etapas de seleção, programação, aquisição, armazenamento, distribuição, utilização: prescrição, dispensação ${ }^{13}$ e nos saberes e práticas da atenção farmacêutica 1,8,9,12,14-16.

As estratégias se deram de forma mista entre instituições públicas e privadas e o estabelecimento de parcerias entre o governo e as farmácias comunitárias privadas. De forma abrangente, a organização dos serviços farmacêuticos se deu por meio de um trabalho em conjunto de várias instituições responsáveis pelo controle, regulamentação, aspectos relacionados a distribuição, fiscalização, entre outros, que envolvem produtos farmacêuticos e a profissão farmacêutica.

O Estado, em conjunto com o seus respectivos Ministérios da Saúde e Economia, designou órgãos responsáveis por fiscalizar e garantir como objetivo principal o acesso ao uso seguro e adequado do medicamento, além de estarem encarregados por toda parte regulamentária que abrange a assistência farmacêutica e suas imputações. Os órgãos que regulam os serviços farmacêuticos, entre as suas diversas atribuições, têm como princípio em comum a garantia do acesso aos medicamentos seguros e adequados, responsáveis por regulamentar, avaliar, autorizar, disciplinar, fiscalizar, verificar todos os procedimentos associados ao setor farmacêutico, seja ele público ou privado, com base na legislação vigente ${ }^{\mathbf{1 7}}$.

As produções foram enfáticas com relação às necessidades logísticas de provimento de equipamentos de proteção individual recomendados para prevenção e higiene, como formulações à base de álcool e máscaras cirúrgicas. Ung ${ }^{9}$ trouxe como uma das medidas adotadas em Macau a familiarização do farmacêutico com as formulações à base de álcool para uma possível produção dentro da farmácia. 
Outras experiências descreveram medidas autorizadas pelas autoridades sanitárias, para evitar o desabastecimento de produtos para higienização de mãos, a exemplo do uso de produtos médicos não aprovados ou usos não aprovados de produtos já existentes ${ }^{9,10}$, ampliação de atribuições profissionais dos farmacêuticos com atividades de educação farmacêutica no contexto de saúde pública ou colaboração com as clínicas de febre do entorno e as instituições Covid-19 quanto ao compartilhamento mútuo de informações durante a transição do tratamento de pacientes em isolamento domiciliar e no rastreamento precoce de casos, a partir da mensuração da temperatura corporal e outros sintomas clínicos como tosse, fadiga e histórico epidemiológico ${ }^{7}$.

Os farmacêuticos estão envolvidos há muito tempo nas ações de saúde pública, incluindo a prevenção através de vacinas de rotina e a garantia do acesso a medicamentos. Por exemplo, o acesso a medicamentos, especialmente durante os surtos de vírus HIV e ebola que datam das décadas de 1980 e 1990, e, mais recentemente, a assistência farmacêutica a idosos e a deficientes, durante a pandemia do H1N1, em 2009, do surto asiático de vírus da influenza aviária, para armazenar e distribuir agentes antivirais e vacinas ${ }^{18,19}$.

Na temporada de gripe, os farmacêuticos e médicos dos EUA colaboram para fazer com que os pacientes recebam tratamento oportuno ${ }^{20}$. Em dois surtos de síndrome respiratória aguda grave (Sars), os farmacêuticos canadenses desempenharam um papel vital na distribuição de medicamentos, informações sobre medicamentos e no apoio direto ao paciente $^{21}$. Sendo a China o primeiro local onde se detectou o Sars-CoV-2, os farmacêuticos chineses, com vasta experiência, responderam rapidamente e executaram uma variedade de estratégias para fornecer atendimento farmacêutico.

No entanto, conforme observado por Gudi e Tiwari ${ }^{22}$, apesar da contagiosidade do H1N1, da virulência do Sars-CoV de 2003 e dos surtos mais recentes da Síndrome Respiratória do Oriente Médio (Mers), esses surtos não estimularam a manutenção de estruturas vibrantes e rapidamente implantáveis para respostas públicas em preparação para a pandemia atual, o que indica a necessidade de melhorar continuamente a capacidade do sistema de saúde de responder a emergências de saúde pública, inclusive no processo de formação na graduação em saúde com foco interprofissional, para ajudar a garantir respostas baseadas em evidências a futuras crises de saúde pública ${ }^{10}$.

Na maioria desses países, as farmácias privadas são amplamente estabelecidas e constituem o ponto inicial e o canal preferido por meio do qual os membros da comunidade poderão obter medicamentos e serviços de saúde para a maioria da população nos países em desenvolvimento ${ }^{23,24}$. Entretanto, o escopo da prática farmacêutica comunitária varia consideravelmente entre os países ${ }^{\mathbf{2 5}, 26}$.

O modelo de assistência farmacêutica predominante nas farmácias comunitárias se constituiu em atenção presencial, educação em saúde com materiais informativos sobre a prevenção da doença, autocuidado e automonitoramento de doenças crônicas, disponibilização de home care farmacêutico e serviços remotos de apoio logístico e cuidado em saúde, incluindo o telemonitoramento da adesão ao tratamento dos pacientes crônicos em uso contínuo de terapia medicamentosa e pacientes com sintomas leves do coronavírus ${ }^{\mathbf{1 , 8}, \mathbf{9}}$. As farmácias se prepararam também para realizar entregas em domicílio, por meio de solicitações por e-mail e aplicativos de medicamentos e insumos farmacêuticos, além de fornecerem serviços regulares de farmácia, no que concerne à segurança de medicamentos 1,8,10,11.

Importante ressaltar que os farmacêuticos desempenham um papel importante na mitigação da escassez emergente de medicamentos relacionada à pandemia. Em primeiro lugar, os farmacêuticos pretendem garantir os medicamentos mais baseados em evidências para os pacientes com Covid-19, a fim de melhorar os resultados ${ }^{27}$. Além disso, os 
farmacêuticos devem dar toda a atenção aos seus conhecimentos farmacológicos e considerar os sintomas e resultados etiológicos do paciente, combinados com a função hepática e renal e os parâmetros farmacocinéticos do medicamento, para formular esquemas de medicamentos individualizados e para melhorar a taxa de cura $^{28}$.

Outra medida frequentemente empregada é definir uma área específica dentro da farmácia para clientes com suspeita de sintomas de Covid-19, a fim de reduzir a transmissão da infecção $0^{29}$.

A preparação dos farmacêuticos da comunidade para um surto de pandemia como a Covid-19 não se limita ao papel clássico da farmácia, mas também deve ser considerada para cobrir o papel de relatar adequadamente casos suspeitos de Covid-19 às autoridades de saúde, monitorando a disseminação da doença e contribuindo para a confiabilidade e validade das figuras nacionais. Um paralelo deve ser traçado com o papel do farmacêutico de relatar reações adversas a medicamentos, especialmente no momento atual da pandemia ${ }^{30}$. Consequentemente, a preparação pandêmica dos farmacêuticos não deve ser negligenciada e deve ser estendida ao conhecimento de procedimentos de notificação.

Entretanto, como em muitas outras áreas da sociedade durante esta pandemia, a Covid-19 destacou e exacerbou muitos dos problemas estruturais já existentes. Na maioria dos países europeus, a tarefa principal do farmacêutico é preparar e distribuir medicamentos prescritos por médicos de família e comunidade ou General Practicioners (GP) ${ }^{31}$. Por outro lado, na Inglaterra, houve uma certa relutância em comprometer fundos adicionais, novos serviços clínicos foram suspensos e houve uma grande escassez no fornecimento de equipamentos de proteção individual, para permitir as interações pessoais que os farmacêuticos comunitários têm com os pacientes ${ }^{32}$.

Tal cenário ainda é um desdobramento das regulamentações publicadas na Europa após a crise econômica de 2008, para os países que foram fortemente afetados pela crise financeira e implementaram mudanças políticas, geralmente com o objetivo de gerar economia brevemente após o surgimento da crise. Neste sentido, foram identificadas medidas políticas direcionadas à redução do financiamento e gastos com medicamentos em Portugal, Grécia, Bélgica, França, República Tcheca, Islândia, Espanha e Alemanha, sendo 22\% das medidas pesquisadas classificadas como de austeridade. Esse cenário pode afetar as decisões, por exemplo, quanto a reembolso por serviços farmacêuticos ambulatoriais frente a uma crise sanitária como a epidemia de Covid-19, que tem a premência de alta transmissibilidade, exigindo decisões políticas rápidas e efetivas ${ }^{33}$.

Um outro aspecto que merece destaque é que, por se enquadrar em serviço de varejo, na maioria dos países, os farmacêuticos que atuam em farmácias comunitárias privadas não estão incluídos na categoria de profissionais de saúde com maior risco de exposição ao coronavírus. Apesar do contato contínuo com usuários e da necessidade de uso de EPIs, em alguns estabelecimentos existem requisitos menos rigorosos para o fornecimento de máscaras e luvas.

Por outro lado, a atuação em serviços farmacêuticos vinculados a equipe multiprofissional em sistemas de saúde de alguns países, como Espanha e Portugal, tem sido apontada como outro aspecto favorável à demanda assistencial na atenção primária à saúde, pois a efetividade do acompanhamento farmacoterapêutico de pacientes com doenças crônicas, por exemplo, diabetes e hipertensão arterial, tem reduzido os custos diretos com assistência médica do Sistema Nacional de Saúde espanhol ${ }^{34}$ e o uso excessivo dos seguros saúde, especialmente entre os idosos ${ }^{35}$.

Para ajudar os médicos a entender e prescrever os medicamentos na lista de medicamentos, os farmacêuticos clínicos criaram um manual racional de uso de medicamentos para o pessoal médico da linha de frente aplicar o tratamento da Covid-19, incluindo uso e dosagem, solventes, precauções, reações adversas a medicamentos e 
ajuste de dose para populações especiais, como mulheres grávidas, crianças, idosos, pacientes em diálise, entre outras situações clínicas ${ }^{36}$. Farmacêuticos nutricionais, como membros da equipe colaborativa multidisciplinar, estabeleceram procedimentos padronizados de terapia nutricional para pacientes graves e críticos com Covid-1937.

Para garantir a assistência farmacêutica em abrigos e de Fangcang, os farmacêuticos da Faculdade de Medicina Tongji, da Universidade de Ciência e Tecnologia de Huazhong, lançaram o serviço Monitoramento Farmacêutico On-line, que é um modelo de serviço farmacêutico on-line, principalmente usando o WeChat, App com dispositivos móveis inteligentes. Cada paciente no hospital-abrigo Fangcang deve responder ao questionário de informações sobre medicamentos na admissão, e as informações sobre medicamentos são carregadas na plataforma de nuvem, $\mathrm{e}$ as perguntas são respondidas imediatamente. O modelo de serviço farmacêutico on-line não apenas reduz efetivamente as chances de infecções adquiridas em hospitais, mas também melhora a eficiência dos serviços de farmácia e obtém orientação profissional oportuna e eficaz sobre medicamentos para pacientes durante todo o processo ${ }^{38,39}$.

No que concerne à continuidade e a longitudinalidade dos cuidados, entre as atividades adequadas para responder às necessidades dos pacientes, alguns estudos citam a inclusão de trabalho remoto. Em um estudo realizado em Bangladesh, onde os serviços de saúde estão se concentrando de maneira incomum em uma pequena fração de pacientes críticos e dispendiosos para a saúde, a telefarmácia inclui aconselhamento ao paciente, revisão de medicamentos e revisão de prescrição por um farmacêutico qualificado para os pacientes residentes em áreas distantes das farmácias ${ }^{40}$.

Além disso, os autores ${ }^{15}$ reforçaram a necessidade de fomentar os comportamentos sociais necessários na fase de mitigação da doença aplicando o modelo de crenças em saúde, por meio da análise dos benefícios percebidos para a ação, as barreiras percebidas para a ação e a capacidade percebida da pessoa para agir. As construções do modelo podem ser aplicadas a pacientes de farmácia comunitária que podem apresentar ansiedade, incerteza, ceticismo ou apreensão com relação aos comportamentos de mitigação da Covid-19. As informações são subsidiadas pelos antecedentes e experiências de um indivíduo. Assim, o farmacêutico comunitário deve ter em mente as características demográficas do paciente, status socioeconômico e base de conhecimento possíveis pela prática clínica contínua.

Outra emergência igualmente premente e paralela à crise sanitária é a sobrecarga de informações sobre medicamentos e informações incorretas em torno da Covid-19. A OMS descreveu que o surto e a resposta à Covid-19 'foram acompanhados por um enorme infodêmico - uma abundância excessiva de informações'. O 'infodêmico' refere-se aos perigos de um volume excessivo de informações com relação à Covid-19 (incluindo medidas ou curas de prevenção falsa) que suscitam preocupações pelo público em distinguir fato de ficção e por agências governamentais conduzirem políticas baseadas em evidências. À medida que a Covid-19 se transforma em uma crise de saúde pública global, várias reivindicações infundadas sobre curas e transmissão e/ou exposição se apossaram da Internet e das mídias sociais ${ }^{\mathbf{4 1}}$. [tradução dos autores].

Essas desinformações em diferentes conjunturas constituem uma preocupação adicional, pois representam um sério desafio no enfrentamento da pandemia ${ }^{41}$. Portanto, o farmacêutico pode atuar disseminando informações baseadas em evidências científicas sobre a situação local e o progresso geral da infecção e contribuir para a educação em saúde sobre as medidas preventivas do surto de 2019 (CoV) e o reconhecimento precoce dos sintomas.

No quesito das políticas de atenção farmacêutica e assistência farmacêutica 
reestruturadas para esse contexto, destacam-se o uso massivo das tecnologias e do acesso remoto, por meio de websites, telefone e aplicativos móveis como canais para consultas e orientações farmacêuticas ${ }^{\mathbf{8}, \mathbf{9}, 1, \mathbf{1 2}, \mathbf{4 2}, \mathbf{4 3}}$. Carico Jr., Sheppard e Thomas ${ }^{\mathbf{1 5}}$ debateram que, em vez de focarem em medicamentos, como de costume, as consultas farmacêuticas durante a epidemia referem-se, também, ao conhecimento de prevenção da Covid-19, como seleção de máscara e sinais e sintomas e transmissibilidade, reforçando comportamentos que limitam a propagação da pandemia, incluindo distanciamento social e uso de telefonemas ou videoconferência para obter cuidado em saúde, quando possível.

Na situação de surto pandêmico, entre as recomendações de organização da Atenção Primária à Saúde na Austrália, foi incentivado o uso de prescrição eletrônica por meio de um sistema em que os prescritores poderão gerar uma prescrição eletrônica que os pacientes poderão compartilhar com uma farmácia por meio de um token de código de barras QR (Quick Response) exclusivo, enviado por um aplicativo, serviços de mensagens de telefone móvel ou e-mail, que interligava as farmácias comunitárias aos serviços de saúde e desburocratizava o acesso aos medicamentos durante a pandemia ${ }^{37}$. Na França e em Portugal, os farmacêuticos foram autorizados a repetir a administração de medicamentos prescritos para pacientes com condições crônicas, para reduzir a necessidade de consultas médicas e liberar recursos ${ }^{44}$.

Ademais, à medida que a pandemia continua a se estender, a demanda generalizada de médicos levou ao adiamento de revisões de rotina e consultas para pacientes com doenças crônicas. Contudo, ante a necessidade de adesão e continuidade do tratamento de pacientes com doenças crônicas para obter resultados terapêuticos adequados, especialmente durante essa pandemia de Covid-19, os pacientes podem procurar os farmacêuticos comunitários que podem julgar a necessidade de fornecer os medicamentos ${ }^{16}$. Por outro lado, farmácias localizadas nos bairros e drogarias não estão articuladas com o sistema de saúde, portanto, é necessário conectá-las ao processo de assistência médica implementado para atender aos possíveis casos de Covid-1914.

Além disso, uma recomendação frequente tem sido a autorização para testar os pacientes contemplando serviços ampliados fornecidos por farmacêuticos. Exemplos específicos incluem a provisão de testes para pacientes individuais, bem como o tratamento e a vacinação para uma comunidade ou população de pacientes, especialmente aqueles em acesso limitado ou disponibilizado em áreas de maior vulnerabilidade da população.

No cenário da atenção farmacêutica no enfrentamento à Covid-19, o farmacêutico atua com o foco na garantia do uso racional de medicamentos, principalmente para os usuários com doenças crônicas e que se caracterizam como grupo de risco ${ }^{8,9,15,16}$, vacinação e capacitação para imunizar a população quando estiver disponível a vacina para a Covid-19 e na testagem rápida da comunidade, evitando o colapso do sistema de saúde ${ }^{10,11}$ e o desenvolvimento de um fluxograma de sinais e sintomas com tomadas de decisões ${ }^{\mathbf{1 4}}$.

\section{Lições aprendidas}

A produção na área vem sendo publicada em periódicos de relevância para a saúde pública, o que confere importância aos estudos de avaliação para compreensão da dinâmica da prestação do cuidado nos serviços, como análise crítica e como forma de apontar experiências exitosas e rumos a serem seguidos para o enfrentamento da epidemia.

As vulnerabilidades expostas pela pandemia da Covid-19 revelaram a necessidade de reorganização das iniciativas para cuidado às doenças agudas pelos sistemas de saúde. Do mesmo modo, revelaram a premência na ampliação de estratégias para fortalecer as cadeias de suprimentos mais perto de onde os materiais são necessários no mercado interno 
e global. Também houve constatação da necessidade de investimentos em pesquisas direcionadas à produção de vacinas, terapêuticas e métodos não médicos de prevenção vinculados às tecnologias de cuidado em saúde.

Neste sentido, a farmácia comunitária tornou-se um ponto de apoio importante para o enfrentamento da Covid-19, possibilitando acesso à informação e aos cuidados em saúde, uma vez que esses estabelecimentos se encontram dimensionados em toda extensão territorial das regiões de estados, províncias e países. E o farmacêutico torna-se um profissional que pode auxiliar no monitoramento da Covid-19 e de outras doenças crônicas, neste momento em que a sociedade precisa manter o distanciamento social, e a farmácia é um ponto de acesso disponível para a população. A ampliação dos prazos para dispensação de medicamentos de uso crônico e prescrição com assinatura eletrônica também se caracterizou como uma medida efetiva, evitando aglomerações nos serviços de saúde.

A prestação de serviços de farmácia, durante o distanciamento social ou isolamento social, fornecendo aconselhamento aos usuários e às equipes multidisciplinares da linha de frente e garantindo o uso racional de medicamentos durante a epidemia, com o desenvolvimento de serviços farmacêuticos inovadores e remotos, contribui de forma abrangente para a ampliação do cuidado em saúde durante a pandemia.

Uma outra medida importante foi a autorização para realização de testes rápidos para o Sars-CoV-2 realizados por farmacêuticos de farmácia comunitária. Foi uma aposta de alguns países para a detecção em tempo oportuno da doença a partir de protocolos e critérios a serem avaliados por esses profissionais e direcionados às autoridades sanitárias, tendo essa ação a proposta de reduzir o impacto da doença para a população e para os sistemas de saúde, bem como ampliar as práticas de prestação de serviços farmacêuticos à comunidade.

Salienta-se como uma das limitações deste estudo a escassez de estudos relacionados a países europeus e latino-americanos para ampliação da abrangência das atribuições dos serviços farmacêuticos para o enfrentamento da pandemia.

O cenário de crise sanitária mundial evidenciou a necessária inclusão e o uso das tecnologias digitais pelos serviços de saúde para ampliar a resolubilidade dos sistemas de saúde, inclusive os farmacêuticos, tendo em vista que os cuidados domiciliares com apoio da telemedicina para reduzir a exposição à Covid-19 são eficazes, e, particularmente, nas áreas rurais e remotas, podendo ser utilizados com maior abrangência após o término desta pandemia.

\section{Colaboradores}

Prado NMBL (0000-0001-8243-5662)*, Carvalho VN (0000-0002-7456-7099)*, Nunes FG (0000-0003-1653-0842)*, Jesus NN (0000-0003-3370-0639), Santos HLPC (0000-0003-2722-7945)* e Santos AM (00000001-9718-1562)* contribuíram igualmente para a elaboração do manuscrito. 


\section{Referências}

1. Liu S, He GF, Du J, et al. Pharmaceutical emergency guarantee difficulties andc ounter measures for the prevention and control of outbreak of Novel Coronavirus Pneumonia (NCP). Chin J Hosp Pharm. 2020; (40):243-249.

2. World Health Organization. Coronavirus disease (COVID-19) technical guidance: Maintaining Essential Health Services and Systems. [acesso em 2020 jan 19]. Disponível em: https:// www.who.int/emergencies/diseases/novel-coronavirus-2019/technical-guidance-publicati ons?healthtopics $=$ b6 bd $35 \mathrm{a} 3-\mathrm{cf} 4 \mathrm{f}-4851-8 \mathrm{e} 80-$ -85 cb0068335b\&publishingoffices $=$ aeebab07-3d0c-4a24-b6ef-7c11b7139e43\&healthtopics-hidden=true \&publishingoffices-hidden=true.

3. Zhao RS, Yang YH, Yang L, et al. Novel coronavirus control and prevention strategy: pharmaceutical guidance and management strategy. Chin Pharmaceut J. 2020. [acesso em 2020 jan 19]. Disponível em: http://kns.cn ki.net/kcms/detail/11.216 2.R.20200207.0936.002.html.

4. Rutter V, Chan AHY, Tuck C, et al. Weaving the health and pharmaceutical care agenda through the themes of the commonwealth heads of government meeting (CHOGM), London 2018. J Pharm Policy Pract. 2018; 11(1):10.

5. Wang RR, Xu Q, Li L, et al. Pharmacological care strategy for antivirals in patients with COVID-19 complicated by underlying disorders. Chin J Hosp Pharm. 2020; (40):1.

6. Cadogan CA, Hughes CM. On the frontline against COVID19: Community pharmacists' contribution during a public health crisis. Research in Soc. Admin. Pharm. (no prelo), 2020.

7. Moher D, Liberati A, Tetzlaff J, et al. Preferred reporting items for systematic reviews and meta-analyses: the PRISMA statement. PLoS Med. 2009; 6(7):e1000097.
8. Zheng SQ, Yang L, Zhou PX, et al. Recommendations and guidance for providing pharmaceutical care services during COVID-19 pandemic: A China perspective. Res Social Adm Pharm. 2020; 17(1):1819-1824.

9. Ung COL. Community pharmacist in public health emergencies: Quick to action against the coronavirus 2019-nCoV outbreak. Res Social Adm Pharm. 2020; 16(4):583-586.

10. Aruru M, Truong HA, Clark S. Pharmacy Emergency Preparedness and Response (PEPR): a proposed framework for expanding pharmacy professionals' roles and contributions to emergency preparedness and response during the COVID-19 pandemic and beyond. Res Social Adm Pharm. 2020; 17(1):1967-1977.

11. Hedima EW, Adeyemi MS, Ikunaiye NY. Community Pharmacists: On the frontline of health service against COVID-19 in LMICs. Res Social Adm Pharm. 2020; 17(01):1964-1966.

12. Al-Quteimat OM, Amer AMR. Sars-Cov-2 outbreak: How can pharmacists help? Res Social Adm Pharm. 2020; 17(2):480-482.

13. Marin N, Luzia VL, Osorio-de-Castro CGS, et al. Assistência farmacêutica para gerentes municipais. Gerentes Municipais. Rio de Janeiro: OPAS; OMS; 2003.

14. Amariles P, Ledezma-Morales M, Salazar-Ospina A, et al. How to link patients with suspicius COVID-19 to health system from the community pharmacies? A route propouse. Res Social Adm Pharm. 2021; 17(1):1988-1989.

15. Carico Junior RR, Sheppard J, Thomas CB. Community pharmacists and communication in the time of COVID-19: Applying the health belief model. Res Social Adm Pharm. 2020; 2021; 17(1):1984-1987.

16. Kretchy IA, Asiedu-Danso M, Kretchy JP. Medication management and adherence during the COVID-19 pandemic: Perspectives and experiences from 
low-and middle-income countries. Res Social Adm Pharm. 2021; 17(01):2023-2026.

17. Management Sciences for Health. Chapter 6: Pharmaceutical legislation and regulation. Manag Sci Heal. 2012 [acesso em 2020 jan 19]; 6(1):1-16. Disponível em: https://www.mendeley.com/catalogue/ chapter-6-pharmaceutical-legislation-regulation/.

18. Schwerzmann J, Graitcer SB, Jester B, et al. Evaluating the Impact of Pharmacies on Pandemic Influenza Vaccine Administration. Disaster Med Public Health Prep. 2017; 11(5):587-593.

19. Miller S, Patel N, Vadala T, et al. Defining the pharmacist role in the pandemic outbreak of novel HiN influenza. J Am Pharm Assoc. 2012; 52(6):763-7.

20. ME Klepser, DG Klepser, AM Dering-Anderson, et al. Effectiveness of a collaborative medical-pharmaceutical program to manage influenza-like illnesses. J Am Pharmaceut Assoc. 2016; (56):229.

21. TWF Chin, Chant C, Tanzini R, et al. Severe acute respiratory syndrome (SARS): the role of the pharmacist. Farmacoterapia. 2004; 24(6):705-712.

22. Gudi SK, Tiwari KK. Preparedness and Lessons Learned from the Novel Coronavirus Disease. Int J Occup Environ Med. 2020; 11(2):108 112.

23. World Health Organization. Medicines in health systems: advancing access, affordability and appropriate use. 2014 [acesso em 2021 maio 22]. Disponível em: https://www.who.int/alliance-hpsr/resources/FR webfinal_vl.pdf.

24. Smith $\mathrm{F}$. The quality of private pharmacy services in low and middle-income countries: a systematic review. Pharm World Sci. 2009; 31(3):351-61.

25. Hughes CM, Hawwa AF, Scullin C, et al. Provision of pharmaceutical care by community pharmacists: a comparison across Europe. Pharm World Sci. 2010; 32(4):472-87.
26. Aly M, García-Cárdenas V, Williams K, et al. A review of international pharmacy-based minor ailment services and proposed service design model. Res Social Adm Pharm. 2018; 14(11):989-998.

27. Badreldin HA, Atallah B. Global drug shortages due to COVID-19: Impact on patient care and mitigation strategies. Res Social Adm Pharm. 2021; 17(1):19461949.

28. Meng L, Qiu F, Sun S. Providing pharmacy services at cabin hospitals at the coronavirus epicenter in China. Int J Clin Pharm. 2020 [acesso em 2021 maio 27]; 42(2):305-308. Disponível em: https://link.springer. com/article/10.1007/s11096-020-01020-5.

29. Bahlol M, Dewey RS. Pandemic preparedness of community pharmacies for COVID-19. Research in Social and Administrative Pharmacy. 2021; 17(1):1888-1896.

30. Christian MD, Devereaux AV, Dichter JR, et al. Task Force for Mass Critical Care; Task Force for Mass Critical Care. Introduction and executive summary: care of the critically ill and injured during pandemics and disasters: CHEST consensus statement. Chest. 2014; 146(supl4):8S-34S.

31. Silva ROS, Macêdo LA, Santos GAD Júnior, et al. Pharmacist-participated medication review in different practice settings: Service or intervention? An overview of systematic reviews. PLoS One. 2019; 14(1):e0210312.

32. Bukhari Q, Jameel Y. Will coronavirus pandemic diminish by summer? [acesso em 2020 fev 20]. Disponível em: http://dx.doi.org/10.2139/ssrn.3556998.

33. Vogler S, Zimmermann N, Joncheere K. Policy interventions related to medicines: Survey of measures taken in European countries during 2010-2015. Health Policy. 2016; 120(12):1363-1377.

34. Lopez-Bastida J, Boronat M, Moreno JO, et al. Costs, outcomes and challenges for diabetes care in Spain. Global Health. 2013; (9):17. 
35. Federman AD, Safran DG, Keyhani S, et al. Awareness of pharmaceutical cost-assistance programs among inner-city seniors. Am J Geriatr Pharmacother. 2009; 7(2):117-29.

36. Song Z, Hu Y, Zheng S, et al. Hospital pharmacists' pharmaceutical care for hospitalized patients with COVID-19: Recommendations and guidance from clinical experience. Res Social Adm Pharm. 2021; 17(1):2027-2031.

37. Xu JQ, Li SJ, Diao CD, et al. Clinical nutritional suggestion for patients with severe pneumonia infected with new coronaviruses. Chin J Hosp Pharm. 2020; (40):1-3.

38. Gong WJ, Zhou T, Xu CF, et al. The practice and discussion of online pharmaceutical service mode in square cabin hospital. Chin. J. Hosp. Pharm. 2020; (40):876-879.

39. Meng L, Qiu F, Sun S. Providing pharmacy services at cabin hospitals at the coronavirus epicenter in China. Int J Clin Pharm. 2020; 42(2):305-308.

40. Mohiuddin AK. Prospect of Tele-Pharmacists in Pandemic Situations: Bangladesh Perspective. J Health Care and Research. 2020; 1(2):72-77.

41. Erku DA, Belachew SA, Abrha S, et al. When fear and misinformation go viral: Pharmacists' role in deterring medication misinformation during the infode- mic' surrounding COVID-19. Research in Social and Administrative Pharmacy. 2020 [acesso em 2021 maio 27]; 17(1):1954-1963. Disponível em: https://www.ncbi. nlm.nih.gov/pmc/articles/PMC7252082/.

42. Elson EC, Oermann C, Duehlmeyer S, et al. Use of telemedicine to provide clinical pharmacy services during the SARS-CoV-2 pandemic. Am J Health Syst Pharm. 2020; 77(13):1005-1006.

43. US Department of Health and Human Services. Notification of the application power of remote telehealth communications during the public health emergency nationwide COVID-19. [acesso em 2020 mar 26]. Disponível em: https://www.hhs.gov/hipaa/for-professionals/special-topics/emergency-preparedness/notification-enforcement-discretion-telehealth/index.html.

44. International Pharmaceutical Federation. Information and interim guidelines for pharmacists and the pharmacy workforce. [acesso em 2021 maio 22]. Disponível em: https://www.fip.org/files/content/priority-areas/coronavirus/FIP_Webinar_Coronavirus_ response_20200210.pdf.

Recebido em 28/05/2020

Aprovado em 19/02/2021

Conflito de interesses: inexistente

Suporte financeiro: não houve 